\title{
A differentiable characterization of local contractions on Banach spaces
}

\section{Andreas Hefti*}

\author{
"Correspondence: heft@zhaw.ch \\ Department of Economics, \\ University of Zurich, \\ Bluemlisalpstrasse 10, Zurich, \\ Switzerland \\ School of Management and Law, \\ Zurich University of Applied \\ Sciences, Gertrudstrasse 15, \\ Winterthur, Switzerland
}

\begin{abstract}
This note provides a differentiable characterization of local contractions on an arbitrary Banach space. As a corollary, a refinement to Ostrowski's sufficient condition for local convergence in finite spaces is obtained, which applies to many models, e.g. in economics, ecology or game theory, where one has an interest in fixed point iterations and local stability of discrete dynamic processes. We show that for the local contraction property to hold, continuity of the derivative at the fixed point is indispensable.
\end{abstract}

MSC: 47A45; 39A30; 47A11

Keywords: contraction mapping; spectral radius; attractive fixed point; local stability; difference equations

\section{Introduction}

Let $X$ be a finite-dimensional Banach space, $W \subset X$, and $\phi: W \rightarrow X$ a function. It is well known that if $x^{*}$ is a (Frechet) differentiable fixed point (FP) of $\phi$ and the spectral radius of the Jacobian satisfies $\rho\left(\partial \phi\left(x^{*}\right)\right)<1$, then the iteration

$$
x^{t+1}=\phi\left(x^{t}\right)
$$

converges locally to $x^{*}$ ([1], Chapter 22, p.151). Conversely, if (1) converges locally to $x^{*}$, it must be the case that $\rho\left(\partial \phi\left(x^{*}\right)\right) \leq 1$. In this note we prove a refinement to this result for arbitrary Banach spaces. The result readily applies to finite-dimensional applications of fixed point iterations in many fields, mainly in relation to a set of difference equations but, to the best of our knowledge, has been previously overlooked.

We show that if the derivative $\partial \phi$ is continuous at $x^{*}$, then $\phi$ induces a local contraction at $x^{*}$ if and only if there exists an operator norm $\|\cdot\|_{|\cdot|}$ (in the finite case: a matrix norm) such that $\left\|\partial \phi\left(x^{*}\right)\right\|_{|\cdot|}<1$ or, equivalently, $\rho\left(\partial \phi\left(x^{*}\right)\right)<1$. The 'if' part means that if $\rho\left(\partial \phi\left(x^{*}\right)\right)<1$, then not only does the iterative sequence $\left\{x^{t}\right\}$ given by (1) converge locally to $x^{*}$, but in fact an entire neighborhood $U$ of $x^{*}$ contracts to $\left\{x^{*}\right\}$ by repeated application of $f$ on $U$. Continuity of $\partial \phi$ at $x^{*}$ is indispensable for this result already in the finite case, which is demonstrated in an example. The 'only if' part implies that if (1) converges locally but $\partial \phi\left(x^{*}\right)=1$, then $\phi$ cannot be a local contraction. While local convergence of (1) is weaker than the local contraction property, our result is practically relevant because for many applications, e.g. in evolutionary biology, economics or game theory, one is interested in the

(c) 2015 Hefti. This article is distributed under the terms of the Creative Commons Attribution 4.0 International License (http://creativecommons.org/licenses/by/4.0/), which permits unrestricted use, distribution, and reproduction in any medium, provided you give appropriate credit to the original author(s) and the source, provide a link to the Creative Commons license, and indicate if changes were made. 
local stability of a discrete process (1) at some FP of $\phi$, where $\phi$ typically is continuously differentiable at such FP, and the knife-edge case $\rho\left(\partial \phi\left(x^{*}\right)\right)=1$ is not 'generic'. It therefore makes sense to define a FP $x^{*}$ of $\phi$ to be locally contraction-stable, ${ }^{\mathrm{b}}$ if $\phi$ is differentiable around $x^{*}, \partial \phi$ is continuous at $x^{*}$ and $\rho\left(\partial \phi\left(x^{*}\right)\right)<1$.

We first provide a differentiable characterization for a mapping between arbitrary Banach spaces to be a contraction. ${ }^{\mathrm{c}}$ This serves as a useful lemma from which we derive a characterization of the local contraction property. Further, we clarify the natural connection of the local contraction property to the linearization of $\phi$ at $x^{*}$, and show that continuity of $\partial \phi$ at $x^{*}$ is generally indispensable for the local contraction property to hold at $x^{*}$.

\section{A differentiable characterization of contraction mappings}

Let $\left(X,|\cdot|_{X}\right),\left(Y,|\cdot|_{Y}\right)$ be two Banach spaces and $W \subset X, V \subset Y$ are non-empty subsets. If $|\cdot|_{X},|\cdot|_{Y}$ are vector norms and $A: X \rightarrow Y$ is linear and continuous, we denote the corresponding operator norm by $\|A\|_{|\cdot|} \equiv \sup _{|v| X=1}|A v|_{Y}$. For $X=Y$ we say that $\|\cdot\|_{|\cdot|}$ is induced by a single norm if $|\cdot|_{X}=|\cdot|_{Y}$. Most relevant to applications is the case where $X$ and $Y$ are finite-dimensional, such that $A$ can be identified by a matrix, and $\|\cdot\|_{|\cdot|}$ is an (induced) matrix norm.

The function $\phi: W \rightarrow V$ is a contraction if there are a $q \in(0,1)$ and norms $|\cdot|_{X},|\cdot|_{Y}$ such that $\left|\phi\left(x^{\prime \prime}\right)-\phi\left(x^{\prime}\right)\right|_{Y} \leq q\left|x^{\prime \prime}-x^{\prime}\right|_{X}$ for any $x^{\prime}, x^{\prime \prime} \in W$. The set of all contractions on $W$ in $V$ is denoted as $\hat{\mathcal{K}}(W, V)$. We first characterize when a differentiable mapping is a (global) contraction on a convex set.

Proposition 1 Let $W \subset X$ be an open, convex set, and suppose that $\phi: W \rightarrow V$ is (Frechet)-differentiable. Then $\phi \in \hat{\mathcal{K}}(W, V)$ if and only if

$$
\sup _{x \in W}\|\partial \phi(x)\|_{|\cdot|}<1
$$

Proof ' $\Rightarrow$ ' Let $q=\sup _{x \in W}\|\partial \phi(x)\|_{|\cdot|}<1$. Because $W$ is open and convex, the mean value theorem implies the following bound for any $x, x^{\prime} \in W$ :

$$
\left|\phi(x)-\phi\left(x^{\prime}\right)\right|_{Y} \leq \sup _{0 \leq t \leq 1}\left\|\partial \phi\left(x+t\left(x^{\prime}-x\right)\right)\right\|_{|\cdot|}\left|x-x^{\prime}\right|_{X} \leq q\left|x-x^{\prime}\right|_{X}
$$

Hence $\phi$ is a contraction on $W$. ' $\Leftarrow$ ' Suppose that there are norms and $q<1$ such that $\left|\phi(x)-\phi\left(x^{\prime}\right)\right|_{Y} \leq q\left|x-x^{\prime}\right|_{X}, \forall x, x^{\prime} \in W$. Take $x \in W$ and $v$ with $|v|_{X}=1$. Then there exists $\varepsilon>0$ such that $x+t v \in W$ for $t \in(-\varepsilon, \varepsilon)$, and $q \geq \frac{|\phi(x+t v)-\phi(x)|_{Y}}{|t|}$ for $t \neq 0$. As $\phi$ is differentiable on $W$ it is also directionally (Gateaux) differentiable, and $q \geq|\partial \phi(x) v|_{Y}$. It follows that $\sup _{x \in W}\|\partial \phi(x)\|_{|\cdot|} \leq q<1$.

Remark 1 If $\bar{W}$ is the closure of $W$ and $\phi: \bar{W} \rightarrow V$ is continuous, and differentiable on $W$, it follows that $\phi \in \hat{\mathcal{K}}(\bar{W}, V)$ if and only if (2) is satisfied.

The most important special case has $X=Y$ and $|\cdot|_{Y}=|\cdot|_{X}$. We denote by $\mathcal{K}(W, V)$ the set of all contractions $\phi: W \rightarrow V, W, V \subset X$, where $|\cdot|_{Y}=|\cdot|_{X} \equiv|\cdot|$. That is, $\phi \in \mathcal{K}(W, V)$ if $\phi$ is a contraction for a single norm $|\cdot|$. Clearly, $\mathcal{K}(W, V) \subset \hat{\mathcal{K}}(W, V)$.

The following is an immediate consequence of the proof of Proposition 1. 
Corollary 1 Let $W, V \subset X, W$ open and convex, and $\phi: W \rightarrow V$ be differentiable. $\phi \in$ $\mathcal{K}(W, V)$ if and only if there is $\|\cdot\|_{|\cdot|}$, induced by a single norm, such that (2) is satisfied. If $\phi: \bar{W} \rightarrow V$ is continuous, and differentiable on $W, \phi \in \mathcal{K}(\bar{W}, V)$ if and only if there is $\|\cdot\|_{1 .}$, induced by a single norm, such that (2) is satisfied.

Remark 2 If $X$ and $Y$ are finite-dimensional, Proposition 1 and Corollary 1 can be restated as: If and only if (2) is verified by a (not necessarily induced) matrix norm $\|\cdot\|$, then $\phi \in \hat{\mathcal{K}}(W, V)$, resp. $\phi \in \mathcal{K}(W, V){ }^{\mathrm{d}}$ However, we can restrict ourselves, without loss of generality, to induced matrix norms, when verifying (2).

A geometric interpretation of Corollary 1 is that $\phi \in \mathcal{K}(W, V)$ if and only if its local rates of change in some normalized direction $v$ is everywhere norm-bounded by $0<q<1$, i.e. if and only if there is $|\cdot|$ and $0<q<1$ such that each directional derivative $\partial \phi(x) v,|v|=1$, satisfies $|\partial \phi(x) v| \leq q$ for any $x \in W$.

\subsection{Local contractions}

A map $\phi: W \rightarrow V, W, V \subset X$, is a local contraction at $x_{0} \in W$ if there is a neighborhood $B=B\left(x_{0}, \delta\right)=\left\{x \in W:\left|x-x_{0}\right|<\delta\right\}$ such that $\left.\phi\right|_{B} \in \mathcal{K}(B, V)$. Further, if $A: X \rightarrow X$ is a bounded linear operator, the spectral radius of $A$ is $\rho(A)=\lim _{n \rightarrow \infty}\left\|A^{n}\right\|_{|\cdot|}^{1 / n}$.

Theorem 1 For $W, V \subset X, W$ open, let $\phi: W \rightarrow V, W, V \subset X$, be differentiable and suppose that $\partial \phi$ is continuous at $x_{0} \in W$. Then $\phi$ is a local contraction at $x_{0}$ if and only if there is an operator norm $\|\cdot\|_{|\cdot|}$ such that

$$
\left\|\partial \phi\left(x_{0}\right)\right\|_{|\cdot|}<1 \quad \text { or equivalently } \quad \rho\left(\partial \phi\left(x_{0}\right)\right)<1 .
$$

Proof ' $\Rightarrow$ ' Let $\sigma(x)=\|\partial \phi(x)\|_{|.|}$. Hence $q \equiv \sigma\left(x_{0}\right)<1$. As $\sigma$ is continuous at $x_{0}$ there is $B=B\left(x_{0}, \delta\right)$ such that $\sup _{x \in B}\|\partial \phi(x)\|_{|\cdot|}<1$, and $\left.\phi\right|_{B} \in \mathcal{K}(B, V)$ follows from Corollary 1 . ' $\Leftarrow$ ' If $\left.\phi\right|_{B} \in \mathcal{K}(B, V)$ for $B=B\left(x_{0}, \delta\right)$, then $\left\|\partial \phi\left(x_{0}\right)\right\|_{|\cdot|}<1$ for some $\|\cdot\|_{|\cdot|}$ by Corollary 1 . Finally, $\rho\left(\partial \phi\left(x_{0}\right)\right)<1$ if and only if $\left\|\partial \phi\left(x_{0}\right)\right\|_{|\cdot|}<1$ for some operator norm follows, because $\forall \varepsilon>0$ there exists an operator norm such that $\rho\left(\partial \phi\left(x_{0}\right)\right) \leq\left\|\partial \phi\left(x_{0}\right)\right\|_{|\cdot|} \leq \rho\left(\partial \phi\left(x_{0}\right)\right)+\varepsilon$ (see [2]).

If the conditions of Theorem 1 are met, and $x_{0}=x^{*}$ is a FP of $\phi$, one can possibly find a neighborhood $B=B\left(x^{*}, \delta\right)$ such that $\left.\phi\right|_{B}$ is a locally forward-invariant contraction, i.e. $\left.\phi\right|_{B} \in \mathcal{K}(B, B)$.

Corollary 2 Let $\phi: W \rightarrow V, W, V \subset X$, be differentiable, $\phi\left(x^{*}\right)=x^{*}$ and $\partial \phi$ is continuous at $x^{*}$. Then $\phi$ is a locally forward-invariant contraction at $x^{*}$ if and only if there is an operator norm $\|\cdot\|_{|\cdot|}$ such that

$$
\left\|\partial \phi\left(x^{*}\right)\right\|_{|\cdot|}<1 \quad \text { or equivalently } \quad \rho\left(\partial \phi\left(x^{*}\right)\right)<1 .
$$

Proof By Theorem 1 it only remains to show that $\phi(B) \subset B$. For $x \in B$ we have $\left|\phi(x)-x^{*}\right|=$ $\left|\phi(x)-\phi\left(x^{*}\right)\right| \leq q\left|x-x^{*}\right|<q \delta<\delta$.

Remark 3 In the finite-dimensional case (i.e. $X=\mathbb{R}^{n}$ ) the statement in Corollary 2 holds for some matrix norm. That is, $\left.\phi\right|_{B} \in \mathcal{K}(B, B)$ if and only if $\left\|\partial \phi\left(x^{*}\right)\right\|<1$ for some matrix norm. 
The local contraction property of a locally continuously differentiable function can be entirely described by the linearization $L_{x^{*}}(x)=\partial \phi\left(x^{*}\right) x+\left(I-\partial \phi\left(x^{*}\right)\right) x^{*}$ of $\phi$ at a FP $x^{*}$.

Corollary 3 Let $\phi: W \rightarrow V, W, V \subset X$, be differentiable, $\phi\left(x^{*}\right)=x^{*}$ and $\partial \phi$ is continuous at $x^{*}$. Then $\phi$ is a local forward-invariant contraction at $x^{*}$ if and only if $L_{x^{*}} \in \mathcal{K}(X, X)$.

Proof ' $\Rightarrow$ ' If $L_{x^{*}} \in \mathcal{K}(X, X)$, then there is $|\cdot|$ and $q<1$ such that $\left|\partial \phi\left(x^{*}\right) v\right| \leq q|v|, v \in X$. Hence also $\left\|\partial \phi\left(x^{*}\right)\right\|_{|\cdot|} \leq q$, and the claim follows from Corollary 2 . ' $\Leftarrow$ ' Equation (4) implies that $\left\|\partial \phi\left(x^{*}\right)\right\|_{|\cdot|} \equiv q<1$ for some $|\cdot|$. Hence $\left|L\left(x^{\prime}\right)-L(x)\right|=\left|\partial \phi\left(x^{*}\right)\left(x^{\prime}-x\right)\right| \leq$ $\left\|\partial \phi\left(x^{*}\right)\right\|_{|\cdot|}\left|x^{\prime}-x\right|=q\left|x^{\prime}-x\right|$, for any $x, x^{\prime} \in X$.

If $\phi$ is differentiable at a FP $x^{*}$, then $\phi(x)-x^{*}-\partial \phi\left(x^{*}\right)\left(x-x^{*}\right)=o\left(\left|x-x^{*}\right|\right), x \rightarrow x^{*}$, and it follows from differentiability that if $\rho\left(\partial \phi\left(x^{*}\right)\right)<1, \exists \delta>0, q<1$, and a norm $|\cdot|$ such that

$$
\left|\phi(x)-x^{*}\right| \leq q\left|x-x^{*}\right|, \quad x \in B\left(x^{*}, \delta\right) .
$$

Consequently, (1) converges locally to $x^{*}$. " We now show that neither $\rho\left(\partial \phi\left(x^{*}\right)\right)<1$ nor (5) imply that $\phi$ has the local contraction property at $x^{*}$ without continuity of $\partial \phi$ at $x^{*}$.

Consider the function $\phi: \mathbb{R} \rightarrow \mathbb{R}$, defined by

$$
\phi(x)= \begin{cases}x^{2} \operatorname{Sin}\left(\frac{1}{x}\right), & x \neq 0 \\ 0, & x=0\end{cases}
$$

This function has a FP $x^{*}=0$ and is everywhere differentiable, but $\partial \phi$ is not continuous at 0 . Because $\rho\left(\phi^{\prime}(0)\right)=0,(5)$ is satisfied, and the iteration (1) converges locally to 0 . Nevertheless, $\phi$ is not a local contraction at 0 .

To see this, note first that for any neighborhood $B(0, \delta)$ there exists $x \in B$ such that $\phi^{\prime}(x)=1$. Differentiation yields

$$
\phi^{\prime}(x)=2 x \operatorname{Sin}\left(\frac{1}{x}\right)-\operatorname{Cos}\left(\frac{1}{x}\right), \quad x \neq 0,
$$

and $\phi^{\prime}(x)=1$ requires that $2 x \operatorname{Sin}\left(\frac{1}{x}\right)=1+\operatorname{Cos}\left(\frac{1}{x}\right)$. But for any $n_{0} \in \mathbb{N}$ the periodicity of sine and cosine imply that $\operatorname{Sin}\left(\left(1+2 n_{0}\right) \pi\right)=0$ and $\operatorname{Cos}\left(\left(1+2 n_{0}\right) \pi\right)=-1$. It follows that for any given $n_{0} \in \mathbb{N}$ the unique $x_{0}$ that verifies $\frac{1}{x_{0}}=\left(1+2 n_{0}\right) \pi$ also has $\operatorname{Cos}\left(\frac{1}{x_{0}}\right)=-1$ and $\operatorname{Sin}\left(\frac{1}{x_{0}}\right)=0$, and thus $\phi^{\prime}\left(x_{0}\right)=1$. Further, for $n \rightarrow \infty$ the equation $\frac{1}{x_{n}}=(1+2 n) \pi$ defines a strictly decreasing sequence $\left\{x_{n}\right\}$ with $\phi^{\prime}\left(x_{n}\right)=1$ for each $n \in \mathbb{N}$ and limit zero. ${ }^{\mathrm{f}}$ This assures the existence of $x_{0} \in B$ such that $\phi^{\prime}\left(x_{0}\right)=-1$. Now, suppose that $\phi$ is a local contraction at 0 , i.e. there is a neighborhood $B(0, \delta)$ such that $\left.\phi\right|_{B} \in \mathcal{K}(B, \mathbb{R})$. Because $\phi$ is differentiable it then follows from Corollary 1 that there exists $\|\cdot\|_{|\cdot|}$ induced by a single norm such that $\sup _{x \in B}\left\|\phi^{\prime}(x)\right\|_{|\cdot|}<1$. According to the previous argument there exists $x_{0} \in B$ such that $\phi^{\prime}\left(x_{0}\right)=\rho\left(\phi^{\prime}\left(x_{0}\right)\right)=1$, which together with the fact that $\rho\left(\phi^{\prime}(x)\right) \leq\left\|\phi^{\prime}(x)\right\|_{|\cdot|}<1$ for any $x \in B$ yields a contradiction. 
Author's contributions

The author conducted by himself all the research related to this article. In particular he carried out all proofs, wrote the manuscript and read and approved the final version.

\section{Acknowledgements}

I am very grateful to two anonymous referees for their clear and useful suggestions and comments as well as to Ines Brunner for her continuing support and input.

\section{Endnotes}

a Illustration: In game theory the function $\phi$ would correspond to the joint best-reply function of all players, where it is the standard case that $\phi$ is continuously differentiable at interior FP of $\phi$, and $\rho\left(\partial \phi\left(x^{*}\right)\right)=1$ is not robust to small perturbations of the game (e.g. [3]).

b See [4] for a recent application in game theory

c We obtain as a corollary a result by Kantorovich and Akilov, who show that if $\left(X,|\cdot|_{X}\right)$ is a fixed Banach space, $W \subset X$, $\phi: W \rightarrow X$ is differentiable, $W_{0} \subset W$ is closed and convex, $\phi\left(W_{0}\right) \subset W$ and (2) is satisfied, then $\phi$ is a contraction on $W_{0}([5]$, Chapter 17, p.501)

d This holds because induced matrix norms are uniformly minimal (see e.g. [6], Chapter 5, p.302).

e This essentially is Ostrowski's proof.

$\mathrm{f}$ The choice of the norm is irrelevant here because we are in a finite-dimensional space.

Received: 18 April 2015 Accepted: 11 June 2015 Published online: 07 July 2015

\section{References}

1. Ostrowski, A: Solution of Equations in Euclidean and Banach Spaces. Academic Press, New York (1973)

2. Holmes, RB: A formula for the spectral radius of an operator. Am. Math. Mon. 75(2), 163-166 (1968)

3. Moulin, H: Dominance solvability and Cournot stability. Math. Soc. Sci. 7, 83-102 (1984)

4. Hefti, $A$ : On the relationship between uniqueness and stability in sum-aggregative, symmetric and general games. ECON Working paper (2015)

5. Kantorovic, L, Akilov, G: Functional Analysis. Akademie Verlag, Berlin (1982)

6. Horn, RA, Johnson, CR: Matrix Analysis. Cambridge University Press, Cambridge (1985)

\section{Submit your manuscript to a SpringerOpen ${ }^{\circ}$ journal and benefit from:}

- Convenient online submission

Rigorous peer review

- Immediate publication on acceptance

- Open access: articles freely available online

- High visibility within the field

- Retaining the copyright to your article 